\title{
Complementary RNA and Protein Profiling Identifies Iron as a Key Regulator of Mitochondrial Biogenesis
}

\author{
Jarred W. Rensvold ${ }^{1}$, Shao-En Ong ${ }^{2}$, Athavi Jeevananthan ${ }^{1}$, Steven A. Carr ${ }^{3}$, Vamsi K. \\ Mootha $^{3,4}$, and David J. Pagliarini 1, $^{, *}$ \\ ${ }^{1}$ Department of Biochemistry, University of Wisconsin-Madison, Madison, WI 53706, USA \\ ${ }^{2}$ Department of Pharmacology, University of Washington, Seattle, WA 98195, USA \\ ${ }^{3}$ Broad Institute of MIT and Harvard, Cambridge, MA 02142, USA \\ ${ }^{4}$ Departments of Systems Biology and Medicine, Harvard Medical School, Boston, MA 02446, \\ USA
}

\section{Summary}

Mitochondria are centers of metabolism and signaling whose content and function must adapt to changing cellular environments. The biological signals that initiate mitochondrial restructuring and the cellular processes that drive this adaptive response are largely obscure. To better define these systems, we performed matched quantitative genomic and proteomic analyses of mouse muscle cells as they performed mitochondrial biogenesis. We find that proteins involved in cellular iron homeostasis are highly coordinated with this process and that depletion of cellular iron results in a rapid, dose-dependent decrease of select mitochondrial protein levels and oxidative capacity. We further show that this process is universal across a broad range of cell types and fully reversed when iron is reintroduced. Collectively, our work reveals that cellular iron is a key regulator of mitochondrial biogenesis, and provides quantitative data sets that can be leveraged to explore posttranscriptional and posttranslational processes that are essential for mitochondrial adaptation.

\section{Introduction}

Mitochondria are ubiquitous organelles that are essential for cellular energy generation and a range of key metabolic pathways. The production of mitochondria-termed mitochondrial biogenesis - is a complex process involving the orchestrated transcription, translation, and import of more than 1,000 proteins encoded by two genomes (Mick et al., 2011; Pagliarini et al., 2008; Scarpulla, 2008; Schmidt et al., 2010). Moreover, these organelles vary considerably in composition across tissues (Mootha et al., 2003a; Pagliarini et al., 2008) and remodel to meet cellular needs (Baltzer et al., 2010; Hock and Kralli, 2009), indicating that the mitochondrial biogenesis program is customizable and responsive to environmental

\footnotetext{
(C) 2013 The Authors

*Correspondence: pagliarini@wisc.edu.

Accession Numbers: The Gene Expression Omnibus accession number for the microarray data reported in this paper is GSE42299. The processed microarray and quantitative proteomic data are also available for download from http://www.pagliarinilab.org/datasets. Supplemental Information: Supplemental Information includes Extended Experimental Procedures, four figures, and two tables and can be found with this article online at http://dx.doi.org/10.1016/j.celrep.2012.11.029.

Licensing Information: This is an open-access article distributed under the terms of the Creative Commons AttributionNonCommercial-No Derivative Works License, which permits non-commercial use, distribution, and reproduction in any medium, provided the original author and source are credited.
} 
conditions. Defects in this process are associated with a range of human disorders, including mitochondrial encephalomyopathy with ragged red fibers (MERRF), type 2 diabetes, and various cancers (Calvo and Mootha, 2010; DiMauro and Schon, 2003; Lowell and Shulman, 2005; Wallace, 2005).

During the past two decades, major progress has been made in deciphering the transcriptional networks that drive mitochondrial biogenesis. Of particular importance was the identification of peroxisome proliferator-activated receptor $\mathrm{\gamma}$, coactivator $1 \mathrm{a}$ (PGC-1a) (Puigserver et al., 1998). PGC-1 $\alpha$ and related coactivators PGC-1 $\beta$ (Kressler et al., 2002; Lin et al., 2002) and PRC (PGC-1 related coactivator) (Andersson and Scarpulla, 2001) coordinate and activate the various transcription factors required for mitochondrial biogenesis (Scarpulla, 2008). Additionally, PGC-1a is activated by exercise, adaptive thermogenesis, changes in cellular redox state, and the availability of nutrients and growth factors (Hock and Kralli, 2009), helping to explain how mitochondrial content is responsive to changing cellular conditions.

Despite significant advancements in our understanding of PGC-1 $\alpha$ and its corresponding transcription factors, important aspects of the cellular control of mitochondrial content remain unclear. These include posttranscriptional processes that control mitochondrial gene expression, mechanisms of active mitochondrial degradation and clearance, and extramitochondrial processes that help coordinate communication between mitochondria and the nucleus (Goldenthal and Marín-García, 2004). Posttranscriptional control mechanisms, such as upstream open reading frames (uORFs) (Calvo et al., 2009), ironresponsive elements (IREs) (Eisenstein and Ross, 2003), and microRNAs (Li et al., 2012), are already known to affect the expression of select mitochondrial genes, and the global discordance between cellular mRNA and protein levels suggests that these mechanisms are likely more widespread (Mootha et al., 2003a). Additionally, macroautophagy (i.e., mitophagy) is emerging as an important mechanism for eliminating damaged mitochondria (Youle and Narendra, 2011). However, identifying additional genes subject to posttranscriptional regulation, and spotlighting cellular processes that help synchronize the mitochondrial biogenesis program, would benefit from matched, cell-wide quantitative data of protein and mRNA abundance, which has largely been lacking.

Here, to produce such a resource, we performed parallel quantitative SILAC (stable isotope labeling by amino acids in cell culture)-based proteomics (Ong and Mann, 2006) and microarray analyses of PGC-1a-induced mitochondrial biogenesis in mouse muscle $\mathrm{C} 2 \mathrm{C} 12$ cells. In doing so, we find that key proteins involved in maintaining cellular iron homeostasis are correlated with mitochondrial biogenesis. We further reveal that depriving various cell types of iron through chelation or active transport leads to a rapid and dosedependent attenuation of mitochondrial transcript and protein levels that is fully reversible within 3-4 days. Together, our work demonstrates that iron deprivation results in an active and coordinated downregulation of mitochondrial gene expression, suggesting that the bioavailability of iron is a key parameter for establishing a set point of cellular mitochondrial activity. Because iron deficiency anemia is the most common nutritional disorder worldwide (McLean et al., 2009), this work has broad implications for understanding mitochondrial dysfunction in human health and disease. Additionally, our data serve as a resource for investigating genes subject to posttranscriptional regulation and for identifying additional auxiliary pathways that might be important for calibrating or modulating the mitochondrial biogenesis program. 


\section{Results and Discussion}

\section{Complementary RNA and Protein Profiling of Mitochondrial Biogenesis}

We sought to better define the mitochondrial biogenesis program in $\mathrm{C} 2 \mathrm{C} 12$ mouse myotubes by performing complementary RNA and protein profiling. To maximize the transcriptional activation of mitochondrial genes, we overexpressed PGC-1a-the predominant transcriptional coactivator that drives mitochondrial biogenesis-more than 200-fold using an adenovirus-mediated delivery system. We chose $\mathrm{C} 2 \mathrm{C} 12$ cells as a model because overexpression of PGC-1 $1 \alpha$ in this cell line is sufficient to cause an approximate doubling of mitochondrial mass in 3 days (Wu et al., 1999). This approach allows us to assess the relative contribution of posttranscriptional mechanisms in regulating mitochondrial gene expression and provides a more complete assessment of the cell-wide proteomic changes that accompany PGC-1a-induced mitochondrial production.

Following PGC-1a overexpression, we tracked changes in cellular mRNA and protein levels using microarrays and quantitative SILAC proteomics (Ong and Mann, 2006), respectively (Figure 1A). Consistent with previous studies, our microarray analyses showed that PGC-1a causes a robust increase in the transcript abundance of nuclear-encoded mitochondrial genes, especially those involved in oxidative phosphorylation (OxPhos) (Figure 1B). Our SILAC data revealed similar results for protein levels: of 442 mitochondrial proteins quantified using at least two unique peptides, 263 significantly increased in abundance (Figure 1C). As expected, PGC-1a-induced mRNA and protein fold changes were largely consistent in the direction of change (Figure S1A). However, the protein and mRNA abundances differed by as much as 9-fold for nuclear-encoded mitochondrial genes, and for 97 of these genes, mRNA abundance was increased, whereas the corresponding protein abundance was decreased (Table S1; Figure S1B). This mRNA:protein discordance reveals that regulation of protein stability or translation is likely to be important for titrating the expression level of select genes during PGC-1a-induced mitochondrial biogenesis. More broadly, these observations reveal the utility of our resource for spotlighting mitochondrial proteins whose expression may be subject to multiple levels of regulation.

\section{Iron Chelation Causes a Pervasive Dampening of Mitochondrial Protein and Transcript Levels}

Our experimental approach also enabled us to investigate peripheral genes and pathways that may be important for the mitochondrial biogenesis program. Interestingly, we noted that proteins involved in regulating cellular iron levels were among the most highly up- and downregulated proteins in our SILAC analyses. The transferrin receptor, which is responsible for transporting transferrin-bound iron into cells, increased more than 4-fold with PGC-1a overexpression (Figure 1C). Reciprocally, the level of ferritin heavy chain, part of the ferritin complex that sequesters cellular iron, was decreased (Figure 1C). These results suggest that iron might be essential for the mitochondrial biogenesis program. Increased cellular iron availability during mitochondrial biogenesis might simply be necessary to accommodate the mitochondrial proteins that contain iron as a cofactor or may occur in anticipation of increased output from the mitochondrial iron-sulfur cluster biogenesis pathway (Lill, 2009; Richardson et al., 2010). However, the magnitude of these changes prompted us to explore whether cellular iron levels might impact the mitochondrial biogenesis program holistically.

To test whether loss of cellular iron is sufficient to induce a restructuring of cellular mitochondrial content, we modified the experimental approach outlined in Figure 1A. Here, in lieu of PGC-1a overexpression, we treated the cells with deferoxamine (DFO), a clinically used cell-permeable iron chelator (Chaston and Richardson, 2003). Strikingly, the 
microarray results revealed that DFO treatment strongly diminished the abundance of mitochondrial transcripts (Figure 1D). Overall, the magnitude of the DFO effect was as robust as the powerful PGC-1a effect and was also most prominent for genes encoding proteins involved in OxPhos (Figures 1E and S1C). Once again, our mRNA and protein measurements were largely consistent in direction and magnitude (Figure S1D); however, there were notable differences in these measurements among the OxPhos complexes. Complexes I and II, each of which contain multiple iron-sulfur clusters, were most strongly affected, with protein levels more decreased than the corresponding mRNA levels (Figure 1F). Conversely, transcripts encoding complex V subunits were decreased, whereas their corresponding protein levels were increased or unchanged (Figure 1F). A recent, large-scale study of protein dynamics found that mitochondrial proteins, including complex I and complex V, have largely similar turnover rates under normal conditions (Price et al., 2010), suggesting that the differences in complex I and complex V subunit levels following iron deprivation likely involve posttranscriptional regulation.

To assess whether cellular iron levels affect PGC-1a-induced mitochondrial biogenesis, we repeated the experimental approach a third time with PGC-1a adenovirus added to cells simultaneously with DFO. Here, the presence of DFO had a pervasive dampening effect on the induction of mitochondrial transcripts by PGC-1a (Figures S1E and S1F). Notably, for many of the same OxPhos subunits highlighted in Figure 1F, transcript expression was increased under these conditions, whereas the corresponding protein levels were decreased (Figures $1 \mathrm{G}$ and $\mathrm{S} 1 \mathrm{G}$ ). This again suggests that for select genes, iron chelation may lead to an active, posttranscriptional reduction of expression, as opposed to merely thwarting the effects of PGC-1a.

Our large-scale gene expression and proteomic data show that iron chelation has an approximately equal and opposite effect to that of PGC-1a, a powerful inducer of mitochondrial biogenesis. To validate these results, we performed immunoblots and realtime quantitative PCR (qPCR) on the same samples used for our proteomics and microarray analyses. Consistent with our SILAC data, we found that OxPhos proteins were significantly decreased by the DFO treatment (Figure 2A). These results also validated that this effect was predominantly limited to mitochondrial proteins because representative endoplasmic reticulum, nuclear, and cytoplasmic markers were unaffected by this treatment (Figure 2A). For qPCR measurements, we chose two nuclearencoded OxPhos genes (Ndufb5, which is part of iron-containing complex I; and Atp5a1, a subunit of iron-free complex V) and one mtDNA-encoded gene ( $C O X 1$, which was not represented on the microarray or captured by mass spectrometry). All three genes were significantly upregulated with PGC-1a overexpression and diminished by DFO treatment (Figure 2B). These PCR results reveal that the iron chelation effect is not limited to nuclear-encoded mitochondrial genes or to genes encoding iron-dependent mitochondrial proteins. Furthermore, our microarray and SILAC data demonstrate that the expression of genes encoding non-mitochondrial irondependent proteins is not diminished by the iron chelation treatment (Figure S1H). Collectively, our data show that acute iron deprivation has a specific and dose-dependent dampening effect on mitochondrial protein expression that exceeds a mere cell-wide loss of iron-dependent proteins and processes.

\section{Iron Chelation Causes a Rapid, Universal, and Dose-Dependent Decrease in Mitochondrial Protein Abundance}

The results above suggest that cells might possess the ability to calibrate their mitochondrial protein levels to the concentration of available iron. To further test this hypothesis, we grew myotubes in the presence of increasing concentrations of DFO. The results show that even $20 \mu \mathrm{M}$ DFO has a marked effect on cytochrome $c$ and complex I levels and that the effect is directly proportional to the concentration of DFO (Figure 2C). To begin to assess the 
universality of the iron deprivation effect, we treated myotubes alongside their undifferentiated myoblast counterparts. Surprisingly, the response in myoblasts was even more robust: after just $24 \mathrm{hr}$ of treatment with DFO, the $8 \mathrm{kDa}$-complex I subunit was nearly completely lost, whereas the level of this protein continued to gradually vanish from myotubes up through $72 \mathrm{hr}$ (Figure S2A). This effect was also evident in a panel of cell lines differing in species and tissue of origin (Figure 2D), suggesting that iron availability might be a universal gauge that cells use to calibrate mitochondrial activity.

To further define the effect of DFO on OxPhos protein abundance in undifferentiated myoblasts, we examined individual subunits from each OxPhos complex using standard SDS-PAGE and fully assembled, native OxPhos complexes using blue native PAGE (BNPAGE). SDS-PAGE revealed that DFO causes a decrease in the abundance of complex I, complex II, and complex IV subunits (Figure 2E). Consistent with our proteomic analysis of differentiated myotubes (Figure 1F), BN-PAGE revealed that DFO most strongly affects the abundance of complex I and complex II (Figure 2F). Moreover, iron chelation also seems to affect supercomplex formation (Figure 2F). A distinguishing feature of complexes I and II among the OxPhos machinery is that they each possess multiple iron-sulfur cluster centers. As such, although many mitochondrial proteins are affected by iron deprivation, this suggests that iron-sulfur clusters might be particularly important for the cellular response to this perturbation.

Our data demonstrate that iron deprivation causes a pervasive, but not complete, reduction in the expression of mitochondrial proteins. This result is consistent with a calibrated remodeling of the mitochondrial proteome, as opposed to total mitochondrial turnover. To further test this, we measured changes in mitochondrial mass from $\mathrm{C} 2 \mathrm{C} 12$ myoblasts using MitoTracker and nonyl acridine orange (NAO) following treatment with $100 \mu \mathrm{M}$ DFO. Consistent with a similar recent study by Yoon et al. (2006), our results revealed either no change or a slight increase in mitochondrial mass after DFO treatment (Figure 2G). Additionally, fluorescence microscopy with MitoTracker staining revealed no obvious changes in mitochondrial mass or morphology after administration of DFO (Figure S2B). Together with the observation that select mitochondrial proteins are not diminished with DFO treatment, including VDAC (voltage-dependent anion channel) (Figures 2A and 2C), these results strongly suggest that the iron chelation effect does not involve complete mitochondrial turnover, as would be expected for a mitophagy process.

\section{Iron-Dependent Mitochondrial Restructuring Is Distinct from Prominent Regulators of Mitochondrial Biogenesis}

Through large-scale and targeted measurements of mRNA and protein abundance, we have found that iron chelation causes a powerful downregulation of mitochondrial protein expression. DFO is a well-characterized and clinically used iron chelator (Chaston and Richardson, 2003); nonetheless, to ensure that our observed effects are not an off-target effect of this drug, we deprived cells of iron through two additional mechanisms. First, we depleted cellular iron stores by overexpressing the iron exporter ferroportin (Nemeth et al., 2004) in human embryonic kidney (HEK) 293 cells (one of the lines tested in Figure 2D). Similar to DFO, ferroportin expression resulted in a loss of cytochrome $c$, the complex IV COXIV subunit, and the complex I $8 \mathrm{kDa}$ subunit (Figure 3A). Both treatments also led to a decrease in ferritin, indicating a reduction in cellular iron levels (Figure S3A). Second, we demonstrated that a comparable effect was achieved with $2,2^{\prime}$-dipyridyl (DP), a structurally and functionally distinct iron chelator (Figure $3 \mathrm{H}$ ). Moreover, because the iron chelation effect is robust in the postmitotic myotubes used in our microarray and proteomics experiments, it is likely independent of the known effect that DFO has on cell proliferation (Yu et al., 2007). 
We next sought to determine whether established regulators of mitochondrial biogenesis could explain this response. First, because of the largely reciprocal effects of DFO and PGC-1a treatments (Figure 1E), we hypothesized that DFO might simply cause a decrease in the expression of PGC-1 $1 \alpha$ or its associated transcription factors. From a detailed time course, we found that the abundance of mitochondrial transcripts (Figures 3B and 3C) and proteins (Figure S3B) begins to decrease around $12 \mathrm{hr}$ and reaches a minimum approximately $24 \mathrm{hr}$ after DFO treatment. As a control, we measured the transcript level of Tfrc, whose expression is regulated by iron levels (Figure S3C). We found that DFO had little effect on the transcript levels of Esrra, Nrf1, or Gabpa (the primary PGC-1a-associated transcription factors) (Figure S3C) and caused a marked increase in the levels of Ppargcla mRNA (Figure 3D). Furthermore, PGC-1 $\alpha^{-/}$cells (Uldry et al., 2006) responded comparably to wild-type cells when deprived of iron (Figure 3E), together indicating that iron deprivation does not affect mitochondrial gene expression by opposing the function of this coactivator. Interestingly, the levels of PGC-1 $\beta$ mRNA (Ppargc 1b) (Figure 3F) and protein (Figure S3B) were decreased following DFO treatment. However, similar to PGC- $1 \mathrm{a}^{-/-}$cells, depletion of PGC-1 $\beta$ expression in primary satellite cells isolated from soleus muscle of PGC-1 $\beta^{\mathrm{f} / \mathrm{f} / \mathrm{MLC}-\mathrm{Cre}}$ mice (Zechner et al., 2010) did not cause diminished levels of mitochondrial markers, nor did it alter the cellular response to DFO treatment (Figure $3 \mathrm{G})$. Together, these results suggest that the observed effects of iron deprivation are independent of PGC-1 $\alpha$ and PGC-1 $\beta$.

Iron chelation is also known to activate the hypoxia response by inhibiting the irondependent hydroxylases that constitutively target the transcription factor HIF-1a (hypoxiainducible factor 1a) for degradation (Schofield and Ratcliffe, 2004), and HIF-1a activation has recently been shown to initiate a loss of mitochondrial mass in renal clear cell carcinoma cells (Zhang et al., 2007). To determine if the loss of mitochondrial proteins observed here is also due to HIF-1a stabilization, we treated $\mathrm{C} 2 \mathrm{C} 12$ myoblasts with the 2-oxoglutarate analog dimethyloxalylglycine (DMOG). The same HIF-1a hydroxylases that require iron also require 2-oxoglutarate as a cofactor and can therefore be inhibited by 2-oxoglutarate analogs even when iron levels are normal (Jaakkola et al., 2001). As seen in Figure 3H, $\mathrm{C} 2 \mathrm{C} 12$ cells treated with the iron chelators DFO or DP responded comparably in their ability to stabilize HIF-1a. Cells treated with DMOG also stabilized HIF-1a but did not affect the OxPhos proteins, suggesting that HIF-1a activation is not sufficient for the iron chelation response. To test whether HIF-1a is necessary for this response, we treated wildtype and HIF-1 $\mathrm{a}^{-/-}$cells with DFO for $24 \mathrm{hr}$. These cells showed the same response as all other cell types tested, regardless of the HIF-1a genotype (Figure 3I). Coupled with the fact that HIF-2a is not expressed in this cell line (data not shown), these experiments reveal that the widespread loss of mitochondrial proteins following iron chelation is a distinct, HIFindependent process. Overall, our data reveal that mere deprivation of iron elicits an effect comparable in magnitude to, but independent of, the most well-established drivers of the mitochondrial biogenesis program.

\section{The Mitochondrial Response to Iron Deprivation Is Reversible}

As noted above, depletion of cellular iron has various known side effects on cellular functions, including inhibition of cell division, initiation of apoptosis, and induction of autophagy (De Domenico et al., 2009; Yu et al., 2007). Therefore, we next sought to determine whether our observed mitochondrial effect represents a true acute metabolic adaptation or merely irreversible cellular damage. To do so, we treated $\mathrm{C} 2 \mathrm{C} 12$ mouse myoblasts with or without DFO and, after $24 \mathrm{hr}$, passaged the cells into fresh, DFO-free media (Figure 4A). We continued to passage the cells every $24 \mathrm{hr}$ and took samples at each time point (Figure 4A). Once again, $24 \mathrm{hr}$ of DFO treatment resulted in the selective loss of mitochondrial markers (Figures 4B and S4A). However, 72-96 hr following the removal of 
DFO, protein (Figure 4B) and transcript (Figure S4A) levels were comparable to those found in their untreated counterparts. To determine whether the recovery of mitochondrial proteins also represents a recovery of mitochondrial function, we profiled mitochondrial respiration using a Seahorse XF Analyzer (Figure 4C). As shown in Figure 4D (and Figure $\mathrm{S} 4 \mathrm{~B}$ ), iron chelation led to a strong decrease in basal oxygen consumption rate (OCR) and spare respiratory capacity (Figure 4E). Because DFO is known to suppress cell proliferation (Yu et al., 2007), we normalized the basal OCR of untreated and DFO-treated cells to cell number. Remarkably, the basal OCR of DFO-treated cells recovered within $48 \mathrm{hr}$ following their passage into DFO-free media (Figure 4D). Interestingly, after this recovery of basal OCR, the DFO-treated cells continued to recover their spare respiratory capacity, which reached untreated levels by day 5 (Figure 4E). Coupling efficiency remained unchanged in the iron-depleted cells, indicating that DFO likely does not damage the integrity of the mitochondrial inner membrane (Figure 4F). Altogether, our results reveal that iron deprivation initiates a reversible and adaptive cellular response that involves remodeling of the mitochondrial proteome and a reduction in mitochondrial respiratory function.

\section{Conclusions}

Mitochondria are vital metabolic organelles that must continually adapt to changing external environments and cellular needs. To better define this adaptive process, we performed extensive, matched microarray and quantitative proteomic analyses of mouse muscle cells under a variety of conditions. In doing so, we created a robust resource that can be mined to discover proteins whose expression levels are affected by posttranscriptional regulation and auxiliary cellular processes that both sense the need for and drive mitochondrial restructuring. We leveraged this resource to find that proteins involved in cellular iron homeostasis are coordinated with mitochondrial biogenesis and have shown that depriving cells of iron through a variety of mechanisms results in a rapid downregulation of mitochondrial protein levels and oxidative capacity. We further demonstrated that this drastic effect occurs in a wide range of cell types and that it is fully reversible within 2-3 days following reintroduction of iron. Last, we have shown that this process is independent of the well-established PGC-1 $\alpha$-, PGC-1 $\beta$-, and HIF-1a-driven mitochondrial biogenesis programs. Because iron deficiency is the world's number one nutritional deficiency, affecting approximately $25 \%$ of the population (McLean et al., 2009), our work could have significant implications for human health and disease. Our extensive, freely available mRNA and protein profiling data sets will serve as a rich resource for further exploring the cellular response to acute metabolic stress and the roles of transcriptional and posttranscriptional processes important for mitochondrial biogenesis.

\section{Experimental Procedures}

\section{Cell Culture}

Brown preadipocytes were maintained in high-glucose DMEM with $20 \%$ FBS and $1 \times$ PS (Invitrogen) at $37^{\circ} \mathrm{C}$ and $5 \% \mathrm{CO}_{2}$. Primary soleus muscle satellite cells were maintained on rat tail collagen (Invitrogen)-coated plates in Ham's F-10 media (as detailed in the Extended Experimental Procedures). All other cell lines were maintained in high-glucose DMEM with $10 \%$ FBS and $1 \times$ PS. Deferoxamine mesylate salt $(\mathrm{DFO})$, DMOG, and DP were obtained from Sigma-Aldrich. For ferroportin (Fpn-GFP) expression, HEK293FT cells were transfected with $1 \mu \mathrm{g}$ Fpn-GFP (Nemeth et al., 2004) using Lipofectamine LTX (Invitrogen). Myotube differentiation, metabolic labeling, and adenoviral infection were performed using standard procedures (as detailed in the Extended Experimental Procedures). 


\section{Microarray and Mass Spectrometry}

RNA purification, cRNA preparation, and hybridization to Affymetrix 4302.0 arrays were performed according to established methods (Mootha et al., 2003b). Unique Entrez Gene identifiers from the mapped data were selected and sorted by the average GFP probe intensity from the two metabolic-labeling experiments. Data corresponding to these brightest probes that gave present call $(\mathrm{P})$ across all samples were used for analysis $(8,313$ probes total).

Mass spectrometry was performed using standard procedures (as detailed in the Extended Experimental Procedures). For comparison of mRNA and protein abundance, all data were mapped to Entrez Gene identifiers. Gene products were identified as mitochondrial using the MitoCarta inventory (Pagliarini et al., 2008). OxPhos and iron-containing proteins were manually curated from Gene Ontology annotations.

\section{Real-Time qPCR}

Total RNA was purified using an RNeasy Kit (QIAGEN). First-strand cDNA was synthesized from $500 \mathrm{ng}$ RNA using SuperScript III (Invitrogen). Real-time qPCR was performed using predesigned TaqMan Assays or SYBR green-based detection (ABI) with $A c t b$ (microarray samples) or $R p l p O$ as the endogenous control (see the Extended Experimental Procedures for primer sequences).

\section{Immunoblotting and ELISA}

For immunoblot analysis, $15 \mu \mathrm{g}$ of cleared whole-cell lysate, as determined by BCA assay (Pierce), was separated on a 4\%-12\% Bis-Tris Mini Gel (Invitrogen), transferred to PVDF, and probed with primary antibodies (listed in Table S2). For BN-PAGE, $12 \mu \mathrm{g}$ of mitochondrial protein, prepared as previously described by Pello et al. (2008), was separated on a 3\%-12\% NativePAGE Bis-Tris Mini-Gel (Invitrogen), transferred to PVDF, and probed with an OxPhos Blue Native Antibody Cocktail (MitoSciences). For ferritin measurement, 15-35 $\mu \mathrm{g}$ of cleared whole-cell lysate was analyzed by ELISA (Ramco Laboratories) and normalized to total protein concentration, as determined by BCA assay.

\section{Analysis of Mitochondrial Mass}

For determination of mitochondrial mass, $\mathrm{C} 2 \mathrm{C} 12$ myoblasts were seeded at 4,500 cells/well in 96-well microplates and incubated for $24 \mathrm{hr}$. Following DFO treatment, mitochondria were stained with $100 \mathrm{nM}$ MitoTracker Green FM or NAO (Invitrogen) for $30 \mathrm{~min}$. The cells were then washed $2 \times$ with PBS, and fluorescence was measured using a BioTek Synergy 2 Microplate Reader with a 485/20 excitation, 528/20 emission filter set. Fluorescence was normalized to cell number (as described in the Extended Experimental Procedures).

\section{Oxygen Consumption Measurements}

OCR measurements were performed using a Seahorse Biosciences XF96 Extracellular Flux Analyzer as previously described by Nicholls et al. (2010). Briefly, C2C12 myoblasts were seeded at 12,000 cells/well in XF96 microplates (Seahorse Biosciences). After a $24 \mathrm{hr}$ incubation, the growth media were exchanged for XF Assay Medium (Seahorse Biosciences) supplemented with $25 \mathrm{mM}$ glucose (Sigma-Aldrich). OCR measurements were 5 min periods following $3 \mathrm{~min}$ mix periods. Myoblasts were treated by sequential addition of $1 \mu \mathrm{g} / \mathrm{ml}$ oligomycin (Sigma-Aldrich), $300 \mathrm{nM}$ FCCP (Sigma-Aldrich), and $2 \mu \mathrm{M}$ rotenone (MP Biomedicals). Spare respiratory capacity and coupling efficiency were calculated using Seahorse Bioscience instructions (see Figure S4B for details). Basal OCR was normalized to cell number (as described in the Extended Experimental Procedures). 


\section{Statistics}

$\mathrm{p}$ Values were calculated by Student's two-tailed t test, one-way ANOVA with Tukey's post hoc analysis, Spearman's rank correlation test, or $X^{2}$ contingency test as indicated in the figure legends.

\section{Supplementary Material}

Refer to Web version on PubMed Central for supplementary material.

\section{Acknowledgments}

We would like to thank the members of the Eisenstein, Kaplan, V.K.M., and D.J.P. laboratories for helpful discussions and assistance regarding this project. We specifically thank Sarah Calvo and Dan Arlow of the V.K.M. lab for assistance with microarray analyses, Jerry Kaplan and Ivana De Domenico (University of Utah) for providing the ferroportin expression vector, Bruce Spiegelman (Harvard Medical School) for providing the PGC-1 $\mathrm{a}^{-/-}$cells, Daniel Kelly (Sanford-Burnham) for providing the PGC-1 $\mathrm{b}^{\mathrm{f} / \mathrm{f} / \mathrm{MLC}-\mathrm{Cre}}$ cells, Randall Johnson and Alex Weidemann (UCSD) for providing the HIF-1 $a^{-/-}$cells, Eric Shoubridge (McGill) for providing the MCH58 cells, and Kelly Werner of the D.J.P. lab for critical reading of the manuscript. This work was supported by a Searle Scholars Award, a Shaw Scientist Award, and USDA Hatch Award WIS01671 (to D.J.P.), NIH R01GM077465 (to V.K.M.), and NIH Molecular Biosciences Training Grant 5T32GM007215-37 (to J.W.R.).

\section{References}

Andersson U, Scarpulla RC. Pgc-1-related coactivator, a novel, serum-inducible coactivator of nuclear respiratory factor 1-dependent transcription in mammalian cells. Mol Cell Biol. 2001; 21:37383749. [PubMed: 11340167]

Baltzer C, Tiefenböck SK, Frei C. Mitochondria in response to nutrients and nutrient-sensitive pathways. Mitochondrion. 2010; 10:589-597. [PubMed: 20696279]

Calvo SE, Mootha VK. The mitochondrial proteomeand human disease. Annu Rev Genomics Hum Genet. 2010; 11:25-44. [PubMed: 20690818]

Calvo SE, Pagliarini DJ, Mootha VK. Upstream open reading frames cause widespread reduction of protein expression and are polymorphic among humans. Proc Natl Acad Sci USA. 2009; 106:75077512. [PubMed: 19372376]

Chaston TB, Richardson DR. Iron chelators for the treatment of iron overload disease: relationship between structure, redox activity, and toxicity. Am J Hematol. 2003; 73:200-210. [PubMed: 12827659]

De Domenico I, Ward DM, Kaplan J. Specific iron chelators determine the route of ferritin degradation. Blood. 2009; 114:4546-4551. [PubMed: 19671920]

DiMauro S, Schon EA. Mitochondrial respiratory-chain diseases. N Engl J Med. 2003; 348:26562668. [PubMed: 12826641]

Eisenstein RS, Ross KL. Novel roles for iron regulatory proteins in the adaptive response to iron deficiency. J Nutr. 2003; 133(5, Suppl 1):1510S-1516S. [PubMed: 12730455]

Goldenthal MJ, Marín-García J. Mitochondrial signaling pathways: a receiver/integrator organelle. Mol Cell Biochem. 2004; 262:1-16. [PubMed: 15532704]

Hock MB, Kralli A. Transcriptional control of mitochondrial biogenesis and function. Annu Rev Physiol. 2009; 71:177-203. [PubMed: 19575678]

Jaakkola P, Mole DR, Tian YM, Wilson MI, Gielbert J, Gaskell SJ, von Kriegsheim A, Hebestreit HF, Mukherji M, Schofield CJ, et al. Targeting of HIF-alpha tothe von Hippel-Lindau ubiquitylation complex by O2-regulated prolyl hydroxylation. Science. 2001; 292:468-472. [PubMed: 11292861]

Kressler D, Schreiber SN, Knutti D, Kralli A. The PGC-1-related protein PERC is a selective coactivator of estrogen receptor alpha. J Biol Chem. 2002; 277:13918-13925. [PubMed: 11854298]

Li P, Jiao J, Gao G, Prabhakar BS. Control of mitochondrial activity by miRNAs. J Cell Biochem. 2012; 113:1104-1110. [PubMed: 22135235] 
Lill R. Function and biogenesis of iron-sulphur proteins. Nature. 2009; 460:831-838. [PubMed: 19675643]

Lin J, Puigserver P, Donovan J, Tarr P, Spiegelman BM. Peroxisome proliferator-activated receptor gamma coactivator 1beta (PGC-1beta), a novel PGC-1-related transcription coactivator associated with host cell factor. J Biol Chem. 2002; 277:1645-1648. [PubMed: 11733490]

Lowell BB, Shulman GI. Mitochondrial dysfunction and type 2 diabetes. Science. 2005; 307:384-387. [PubMed: 15662004]

McLean E, Cogswell M, Egli I, Wojdyla D, de Benoist B. Worldwide prevalence of anaemia, WHO Vitamin and Mineral Nutrition Information System, 1993-2005. Public Health Nutr. 2009; 12:444-454. [PubMed: 18498676]

Mick DU, Fox TD, Rehling P. Inventory control: cytochrome c oxidase assembly regulates mitochondrial translation. Nat Rev Mol Cell Biol. 2011; 12:14-20. [PubMed: 21179059]

Mootha VK, Bunkenborg J, Olsen JV, Hjerrild M, Wisniewski JR, Stahl E, Bolouri MS, Ray HN, Sihag S, Kamal M, et al. Integrated analysis of protein composition, tissue diversity, and gene regulation in mouse mitochondria. Cell. 2003a; 115:629-640. [PubMed: 14651853]

Mootha VK, Lindgren CM, Eriksson KF, Subramanian A, Sihag S, Lehar J, Puigserver P, Carlsson E, Ridderstråle M, Laurila E, et al. PGC-1alpha-responsive genes involved in oxidative phosphorylation are coordinately downregulated in human diabetes. Nat Genet. 2003b; 34:267273. [PubMed: 12808457]

Nemeth E, Tuttle MS, Powelson J, Vaughn MB, Donovan A, Ward DM, Ganz T, Kaplan J. Hepcidin regulates cellular iron efflux by binding to ferroportin and inducing its internalization. Science. 2004; 306:2090-2093. [PubMed: 15514116]

Nicholls DG, Darley-Usmar VM, Wu M, Jensen PB, Rogers GW, Ferrick DA. Bioenergetic profile experiment using C2C12 myoblast cells. J Vis Exp. 2010; 46:2511. [PubMed: 21189469]

Ong SE, Mann M. A practical recipe for stable isotope labeling by amino acids in cell culture (SILAC). Nat Protoc. 2006; 1:2650-2660. [PubMed: 17406521]

Pagliarini DJ, Calvo SE, Chang B, Sheth SA, Vafai SB, Ong SE, Walford GA, Sugiana C, Boneh A, Chen WK, et al. A mitochondrial protein compendium elucidates complex Idisease biology. Cell. 2008; 134:112-123. [PubMed: 18614015]

Pello R, Martín MA, Carelli V, Nijtmans LG, Achilli A, Pala M, Torroni A, Gómez-Durán A, RuizPesini E, Martinuzzi A, et al. Mitochondrial DNA background modulates the assembly kinetics of OXPHOS complexes in a cellular model of mitochondrial disease. Hum Mol Genet. 2008; 17:4001-4011. [PubMed: 18806273]

Price JC, Guan S, Burlingame A, Prusiner SB, Ghaemmaghami S. Analysis of proteome dynamics in the mouse brain. Proc Natl Acad Sci USA. 2010; 107:14508-14513. [PubMed: 20699386]

Puigserver P, Wu Z, Park CW, Graves R, Wright M, Spiegelman BM. A cold-inducible coactivator of nuclear receptors linked to adaptive thermogenesis. Cell. 1998; 92:829-839. [PubMed: 9529258]

Richardson DR, Lane DJ, Becker EM, Huang ML, Whitnall M, Suryo Rahmanto Y, Sheftel AD, Ponka P. Mitochondrial iron trafficking and the integration of iron metabolism between the mitochondrion and cytosol. Proc Natl Acad Sci USA. 2010; 107:10775-10782. [PubMed: 20495089]

Scarpulla RC. Transcriptional paradigms in mammalian mitochondrial biogenesis and function. Physiol Rev. 2008; 88:611-638. [PubMed: 18391175]

Schmidt O, Pfanner N, Meisinger C. Mitochondrial protein import: from proteomics to functional mechanisms. Nat Rev Mol Cell Biol. 2010; 11:655-667. [PubMed: 20729931]

Schofield CJ, Ratcliffe PJ. Oxygen sensing by HIF hydroxylases. Nat Rev Mol Cell Biol. 2004; 5:343354. [PubMed: 15122348]

Uldry M, Yang W, St-Pierre J, Lin J, Seale P, Spiegelman BM. Complementary action of the PGC-1 coactivators in mitochondrial biogenesis and brown fat differentiation. Cell Metab. 2006; 3:333341. [PubMed: 16679291]

Wallace DC. A mitochondrial paradigm of metabolic and degenerative diseases, aging, and cancer: a dawn for evolutionary medicine. Annu Rev Genet. 2005; 39:359-407. [PubMed: 16285865] 
Wu Z, Puigserver P, Andersson U, Zhang C, Adelmant G, Mootha V, Troy A, Cinti S, Lowell B, Scarpulla RC, Spiegelman BM. Mechanisms controlling mitochondrial biogenesis and respiration through the thermogenic coactivator PGC-1. Cell. 1999; 98:115-124. [PubMed: 10412986]

Yoon YS, Yoon DS, Lim IK, Yoon SH, Chung HY, Rojo M, Malka F, Jou MJ, Martinou JC, Yoon G. Formation of elongated giant mitochondria in DFO-induced cellular senescence: involvement of enhanced fusion process through modulation of Fis1. J Cell Physiol. 2006; 209:468-480. [PubMed: 16883569]

Youle RJ, Narendra DP. Mechanisms of mitophagy. Nat Rev Mol Cell Biol. 2011; 12:9-14. [PubMed: 21179058]

Yu Y, Kovacevic Z, Richardson DR. Tuning cell cycle regulation with an iron key. Cell Cycle. 2007; 6:1982-1994. [PubMed: 17721086]

Zechner C, Lai L, Zechner JF, Geng T, Yan Z, Rumsey JW, Collia D, Chen Z, Wozniak DF, Leone TC, Kelly DP. Total skeletal muscle PGC-1 deficiency uncouples mitochondrial derangements from fiber type determination and insulin sensitivity. Cell Metab. 2010; 12:633-642. [PubMed: 21109195]

Zhang H, Gao P, Fukuda R, Kumar G, Krishnamachary B, Zeller KI, Dang CV, Semenza GL. HIF-1 inhibits mitochondrial biogenesis and cellular respiration in VHL-deficient renal cell carcinoma by repression of C-MYC activity. Cancer Cell. 2007; 11:407-420. [PubMed: 17482131] 

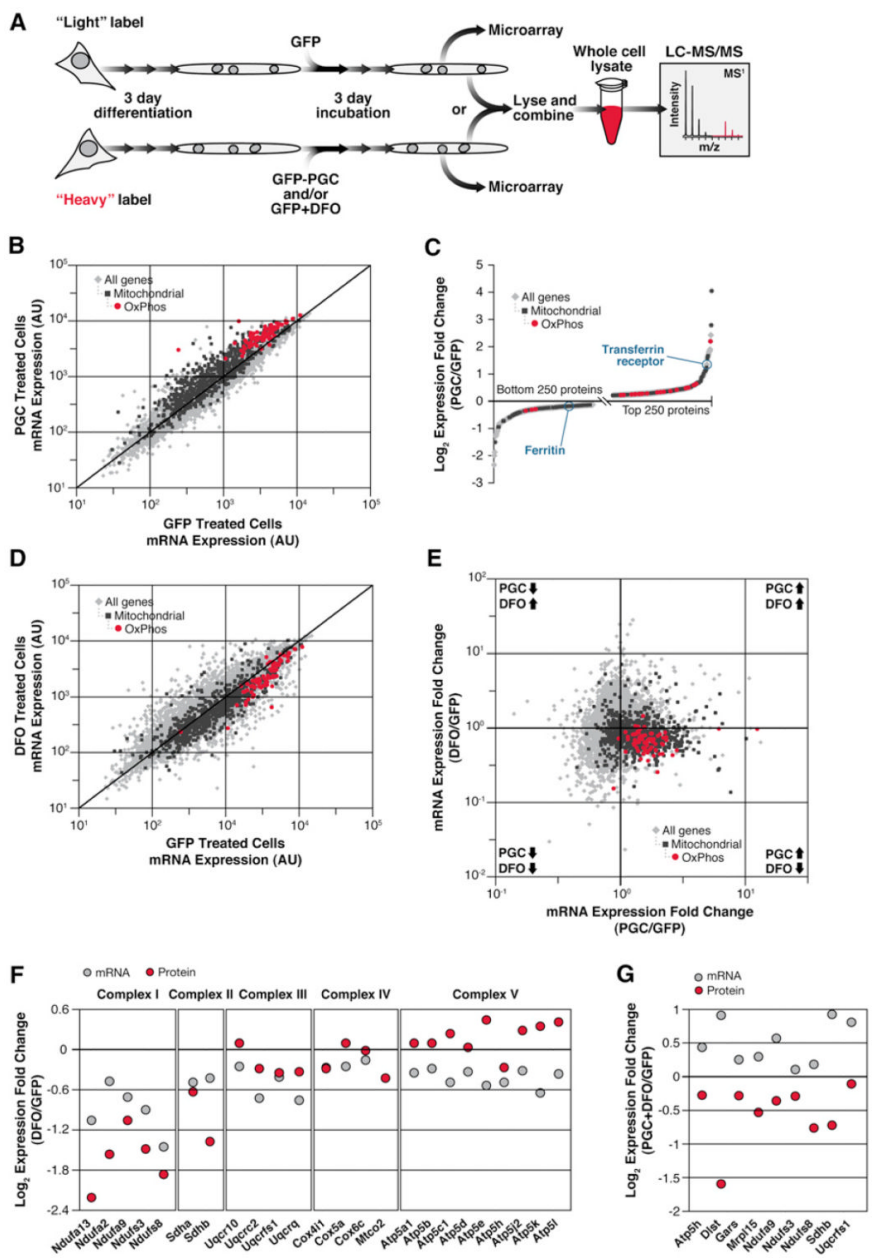

Figure 1. Complementary RNA and Protein Profiling of PGC-1a-Induced Mitochondrial Biogenesis

(A) Experimental workflow for proteomic and microarray analysis of differentiated C2C12 mouse myotubes overexpressing PGC-1a and/or treated with $100 \mu \mathrm{M}$ DFO.

(B) Comparison of mRNA expression in GFP-treated cells and PGC-1a-treated cells (AU, arbitrary units).

(C) Changes in protein expression during PGC-1a overexpression. Proteins are ordered from least to greatest fold change.

(D) Comparison of mRNA expression in GFP-treated cells and DFO-treated cells.

(E) Comparison of PGC-1 $\alpha$ and DFO-induced changes in mRNA expression $(59 \%$ of mitochondrial genes versus $18 \%$ of all genes are in the lower-right quadrant; $p=9.1 \times$ $10^{-222}, \mathrm{X}^{2}$ contingency test).

(F) Comparison of OxPhos mRNA and protein expression during DFO treatment.

(G) Mitochondrial mRNA and protein expression that show discordance during PGC-1a + DFO treatment.

See also Figure S1 and Table S1. 

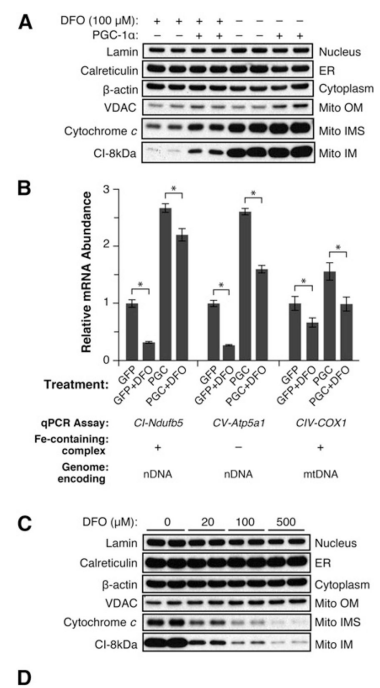

D
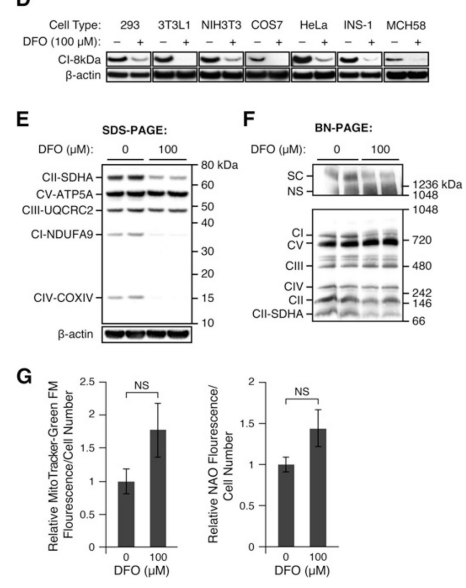

Figure 2. Effect of Iron Deprivation on Nuclear and mtDNA-Encoded Gene Expression

(A) Level of the indicated proteins from samples used in the proteomic analyses as assessed by immunoblotting.

(B) Abundance of the indicated transcripts from samples used in the microarray analyses as detected by real-time qPCR. Data are displayed as mean \pm SD of triplicate measurements $(* \mathrm{p}<0.05$, ANOVA with Tukey's test).

(C) Level of the indicated proteins in $\mathrm{C} 2 \mathrm{C} 12$ myotubes after treatment with a range of DFO concentrations for 3 days as assessed by immunoblotting.

(D) Level of the indicated proteins after DFO treatment for $24 \mathrm{hr}$ in the indicated cell lines as assessed by immunoblotting.

(E) Level of the indicated proteins after DFO treatment for $24 \mathrm{hr}$ in $\mathrm{C} 2 \mathrm{C} 12$ myoblasts as assessed by SDS-PAGE and immunoblotting.

(F) Level of the indicated proteins after DFO treatment for $24 \mathrm{hr}$ in myoblasts as assessed by BN-PAGE and immunoblotting (SC, respiratory supercomplex; NS, nonspecific band). (G) Mitochondrial mass in myoblasts after DFO treatment for $24 \mathrm{hr}$ as assessed by plate reader-based quantitation of MitoTracker Green FM or NAO fluorescence/cell number. Data are displayed as mean \pm SD of triplicate measurements (NS signifies $\mathrm{p} \geq 0.05$, Student's $t$ test).

See also Figure S2. 

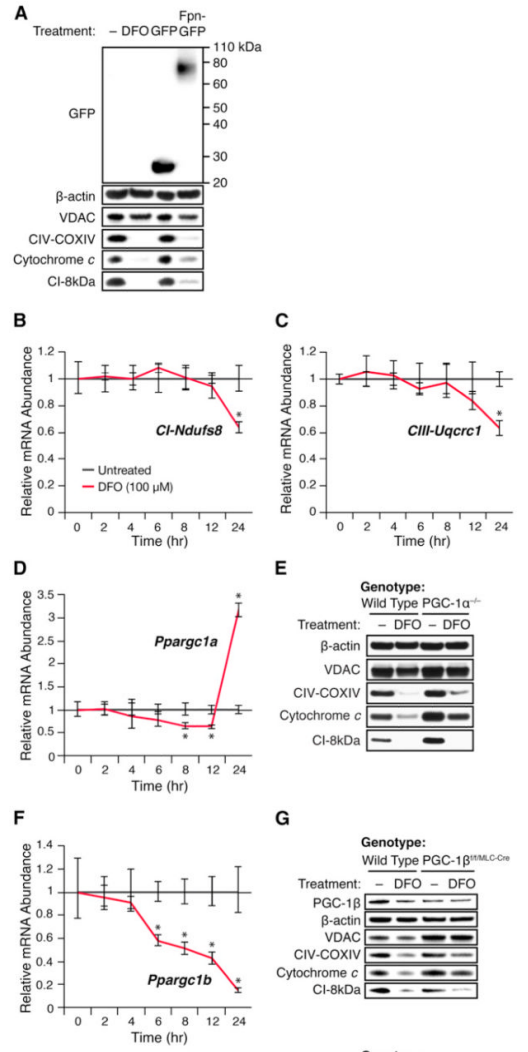

\begin{abstract}
H
\end{abstract}
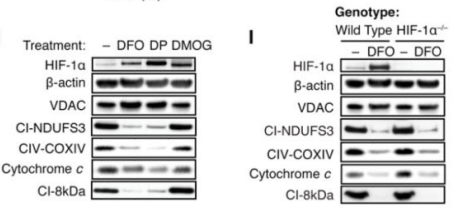

Figure 3. The Mitochondrial Response to Iron Deprivation Is Independent of PGC-1a, PGC-1ß, and HIF-1a

(A-I) Immunoblotting was used to assess protein abundance, and real-time qPCR was used to assess mRNA abundance. Data are displayed as mean \pm SD of triplicate measurements (*p < 0.05, Student's t test). (A) Protein levels in HEK293 cells after $100 \mu \mathrm{M}$ DFO treatment or ferroportin (Fpn-GFP) over-expression for $48 \mathrm{hr}$. Abundance of (B) Ndufs \&, (C) Uqcrc1, and (D) Ppargcla in C2C12 myoblasts following DFO treatment for the indicated times. (E) Level of the indicated proteins in wild-type or PGC- $1 \alpha^{-/-}$brown preadipocytes after 100 $\mu \mathrm{M}$ DFO treatment for $24 \mathrm{hr}$. (F) Abundance of Ppargclb in myoblasts following DFO treatment for the indicated times. $(\mathrm{G})$ Level of the indicated proteins in wild-type or PGC-1 $\mathrm{b}^{\mathrm{f} / \mathrm{f} / \mathrm{MLC}-C r e}$ soleus muscle primary satellite cells after $100 \mu \mathrm{M}$ DFO treatment for $24 \mathrm{hr}$. (H) Level of the indicated proteins after $100 \mu \mathrm{M}$ DFO, $100 \mu \mathrm{M}$ DP, or $500 \mu \mathrm{M}$ DMOG treatment for $24 \mathrm{hr}$ in myoblasts.

(I) Level of the indicated proteins in wild-type or HIF-1 $a^{-/-}$MEFs after $100 \mu \mathrm{M}$ DFO treatment for $24 \mathrm{hr}$.

See also Figure S3. 


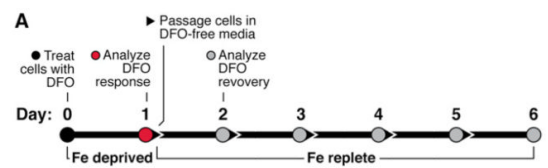

B

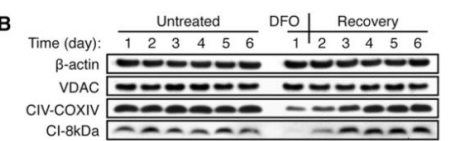

c

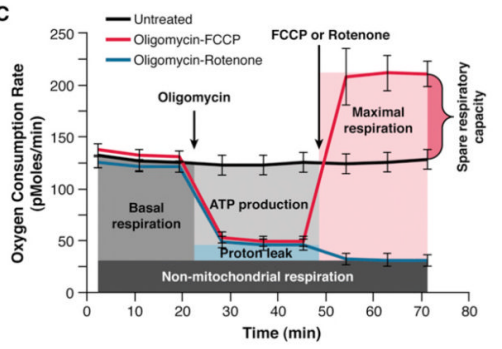

D
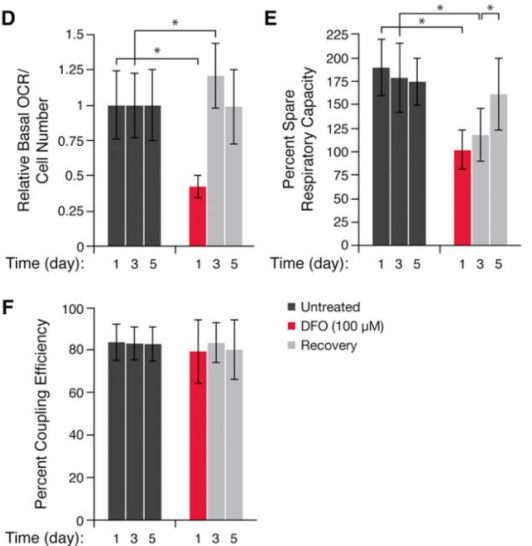

Figure 4. Mitochondrial Gene Expression and Respiratory Function during Iron Deprivation and Recovery

(A) Experimental workflow for the analysis of mitochondrial gene expression and respiration during DFO response and recovery.

(B) Level of the indicated proteins in C2C12 myoblasts treated with $100 \mu \mathrm{M}$ DFO for $24 \mathrm{hr}$ then passaged every $24 \mathrm{hr}$ in DFO-free media as assessed by immunoblotting.

(C) Mitochondrial respiratory profile of untreated myoblasts at day 1 of analysis. Data are displayed as mean \pm SD of 14-16 replicates.

(D-F) Basal OCR (pmol/min)/cell number (D), spare respiratory capacity (E), and coupling efficiency $(\mathrm{F})$ of myoblasts treated with $100 \mu \mathrm{M}$ DFO for $24 \mathrm{hr}$ then passaged every $24 \mathrm{hr}$ in DFO-free media. Data are displayed as mean \pm SD of $14-16$ replicates $(* p<0.05$, Student's $t$ test in D, ANOVA with Tukey's test in E and F).

See also Figure S4. 